\title{
Bio-samtools: Ruby bindings for SAMtools, a library for accessing BAM files containing high-throughput sequence alignments
}

\author{
Ricardo H Ramirez-Gonzalez ${ }^{1}$, Raoul Bonnal ${ }^{2}$, Mario Caccamo ${ }^{1}$ and Daniel MacLean ${ }^{3 *}$
}

\begin{abstract}
Background: The SAMtools utilities comprise a very useful and widely used suite of software for manipulating files and alignments in the SAM and BAM format, used in a wide range of genetic analyses. The SAMtools utilities are implemented in C and provide an API for programmatic access, to help make this functionality available to programmers wishing to develop in the high level Ruby language we have developed bio-samtools, a Ruby binding to the SAMtools library.

Results: The utility of SAMtools is encapsulated in 3 main classes, Bio::DB::Sam, representing the alignment files and providing access to the data in them, Bio::DB::Alignment, representing the individual read alignments inside the files and Bio::DB::Pileup, representing the summarised nucleotides of reads over a single point in the nucleotide sequence to which the reads are aligned.

Conclusions: Bio-samtools is a flexible and easy to use interface that programmers of many levels of experience can use to access information in the popular and common SAM/BAM format.
\end{abstract}

Keywords: Next-generation sequencing, DNA, High, Throughput, Ruby, Bio, SAM, BAM

\section{Background}

High-throughput DNA sequencing in the biological sciences has made it possible for researchers to obtain many millions of sequence reads in single, low-cost experiments. These sequence reads are typically very short compared to the parent genome (reads will usually be in the range of 36 - 200 nucleotides long while genomes are many millions of nucleotides long) and very redundant; many reads may have the same sequence. One widespread use for these sequences is in detecting small differences in the genome sequence of the sample donor, which is achieved by using computational methods to align each short sequence read against a long, reference genome sequence then examining the derived alignments and determining positions at which there are differences. Many programs have been created for alignment including BWA [1], Bowtie [2], SOAP [3], NOVOALIGN [4]

\footnotetext{
*Correspondence: dan.maclean@tsl.ac.uk

${ }^{3}$ The Sainsbury Laboratory, Norwich Research Park, Colney Lane, Norwich, NR4 7UH, UK

Full list of author information is available at the end of the article
}

and BFAST [5], each implementing different algorithms optimised to address different issues with the alignment problem. Most high-throughput alignment programs produce a standard output file in Sequence Alignment/Map format (SAM) [6], a tab-delimited text-based format for describing alignments. The SAMtools utilities comprise a very useful and widely used suite of software for manipulating files and alignments in the SAM format. The large SAM files can be converted to the binary equivalent BAM files a compressed and indexed variant for random access, which vastly facilitates genetic analyses that rely on highthroughput alignment. The SAMtools utilities are implemented in $\mathrm{C}$ and provide an API for programmatic access, for which there are multiple language bindings, notably in Perl [7], Python [8] and Java [9]. Here we describe the Ruby language binding to the SAMtools library, developed for our own work and distributed as a BioRuby plug-in [10]. 


\section{Implementation}

The bio-samtools package is a wrapper around libbam.so (for Linux) and libbam.1.dylib (for Mac OS X), the core shared object library from the SAMtools package. To make it possible for the $\mathrm{C}$ functions in libbam to be called from within Ruby code we have used the Ruby Foreign Function Interface (FFI) [11] package as a bridge between the two languages. The flexible FFI package can programatically load dynamic libraries and bind functions without the need to make changes to Ruby itself or to recompile any extensions, so our package can easily run on standard Ruby interpreters without installation and compilation issues beyond that of the normal Ruby gem installation. Importantly, FFI also has useful methods for managing memory, pointers, structs and binary fields are converted to Ruby boolean variables. A further advantage of using FFI is that the binding is compatible with both the standard Ruby interpreter Matz's Ruby Interpreter (MRI) and the alternative Java implementation of the Ruby language (JRUBY). By wrapping SAMtools in this way the scientist may use the high level easily learned and fast to develop with Ruby that facilitates quick development rather than the native $\mathrm{C}$ of SAMtools. bio-samtools hides the low-level API completely making bio-samtools a useful and easily used tool for working with Next-Generation Sequencing data in BAM files. Each .c library from the SAMtools API is represented by a separate Ruby module mapping the $C$ functions (Figure 1), which are unified in the class Sam.

\section{Bio::DB::Sam}

The main object representing the SAM/BAM file is a Bio::DB::Sam object. Objects of class Sam, have several attributes and methods, summarised in Table 1. Most of the attributes relate to the alignment file type and

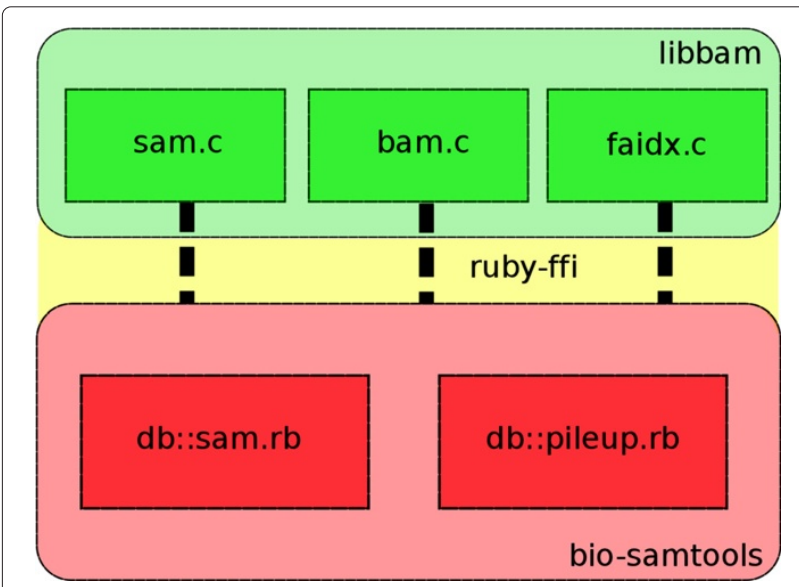

Figure 1 bio-samtools and its relationship to the underlying libbam. Green boxes indicate $C$ source code in libbam, red boxes indicate Ruby files that interact the Ruby FFI represented in yellow. the location of the BAM file in the file system. The BAM file itself is not held in the object or Ruby memory, rather the Ruby wrapping is used to access the information via the C API. The methods of the Sam object can be divided into utility methods that affect the BAM files, (\#sort and \#merge), retrieval methods that return objects of other classes representing individual read alignments (\#fetch, \#fetch_with_function) and summary methods (\#average_coverage, \#chromosome_coverage and \#mpileup,\#index_stats).

\section{Bio::DB::Alignment}

The fetch and fetch_with_function method of the Bio::DB::Sam object return individual alignments one at a time from an iterator. The individual alignments represent a single read and its mapping to the reference and are Bio::DB::Alignment objects, whose attributes are described in Table 2. These attributes are derived directly from the SAM format definition [6]. The fetch_with_function method is distinct from fetch in that it allows the user to pass a Ruby Proc object or a block. These are functionally equivalent to closures in other languages and provide advantages in terms of encapsulation and often speed compared to the standard block based equivalent, advanced Ruby programmers are likely to appreciate this feature.

\section{Bio::DB::Pileup}

The pileup format is a straightforward way of structuring alignments over single positions for the easy identification of genetic polymorphisms, the format has a long history and has been in use in SAMtools for a while. The original 'pileup' function has recently been deprecated and removed in favour of 'mpileup'. The output from mpileup is exactly equivalent to the pileup command called without the -c flag set, that is to say the six column format. The class Pileup can parse the old ten column pileup format if an instance is created manually by passing it a raw line from the pileup file. Calling the mpileup method of a SAM object results in the return of a stream of Pileup [12] objects, which have the attributes and methods listed in Table 3. Some of the attributes are related to the ten column format only. Notably, SAMtools will calculate a consensus base call if asked to return a ten column pileup file, so the Pileup class will use SAMtools consensus call if it is available, otherwise it will call a consensus based on a simple majority count.

\section{Results and discussion}

\section{Using bio-samtools: a brief tutorial}

bio-samtools in use is straightforward, here are a few examples of interacting with BAM files with the package. More information on specific functions is provided in the RubyDoc documentation and in the 
Table 1 Attributes and methods of the Bio::DB::Sam object

\begin{tabular}{ll}
\hline binary & denotes whether this is a binary file \\
\hline compressed & denotes whether this file is compressed \\
\hline sam & path to the reference FASTA file \\
\hline chromosome_coverage & path to the associated BAM file \\
\hline fetch & return Ruby Array of coverage over a region \\
\hline fetch_reference & fetch alignment in a region from a bam file, returning a Ruby Array object \\
\hline fetch_with_function & fetch regions of the reference file returning a String object of the relevant sequence \\
\hline index_stats & fetch all alignments in a region passing in a Ruby Proc object as a callback, returning an iterator \\
\hline merge & get information about reference and number of mapped reads \\
\hline mpileup & merge two or more bam files \\
\hline sort & an iterator that returns Pileup objects representing the reads over a single position \\
\hline
\end{tabular}

files bioruby-samtools/doc/tutorial.html and biorubysamtools/doc/tutorial.pdf. The location of the biosamtools installation folder can be found by typing 'gem which bio-samtools' at the command-line.

Table 2 Attributes of the Bio::DB::Alignment object

\begin{tabular}{ll}
\hline calend & nucleotide position of the end of the alignment \\
\hline cigar & CIGAR string describing the matches/mismatches \\
\hline failed_quality & this read failed the quality threshold \\
\hline is_duplicate & first of a pair \\
\hline is_mapped & this read is a suspected optical or PCR duplicate \\
\hline is_paired & the read was aligned \\
\hline isize & the read is one of a pair \\
\hline mapq & the insert size distance between mapped mates \\
\hline mate_strand & the PHRED scaled mapping quality of the alignment \\
\hline mate_unmapped & the strand of the mate \\
\hline mpos & start position of the mate on the reference \\
\hline pos & start position of the alignments \\
\hline primary & is a primary alignment \\
\hline qlen & read sequence \\
\hline quame & read length \\
\hline query & read name \\
\hline
\end{tabular}

\section{Installation}

bio-samtools is easily installed from a machine with an internet connection and a Ruby installation with the straightforward Gem invocation 'gem install bio-samtools'. bio-samtools automatically downloads the original libbam $\mathrm{C}$ source code and compiles it for Linux or OSX as appropriate. The new version of the library is kept locally to the bio-samtools code to avoid conflicts with other installations of the library.

\section{Loading a BAM file}

A SAM object represents the alignments in the BAM file, and is very straightforward to create, you will need a sorted BAM file, to access the alignments and a reference sequence in FASTA format to use the reference sequence. The object can be created and opened as follows:

require 'bio-samtools'

bam = Bio :: DB :: Sam . new (: bam=>"my_sorted. bam", : fasta $=>^{\prime}$ ref.fasta')

bam. open

bam.close

Opening the file needs only to be done once for multiple operations on it, access to the alignments is random so you don't need to loop over all the entries in the file, as you would with a manual SAM file parse.

\section{Getting summary information}

The length of reference sequences and the number of reads mapped to each can be obtained with the index_stats function. A Hash object, keyed by reference name and with a Hash at each value is returned. The Hash at the value has keys :length, :mapped_reads and :unmapped_reads and values for each of these. The index_stats function wraps the SAMtools idxstats command. 
Table 3 Attributes and methods of the Bio::DB::Pileup object

\begin{tabular}{|c|c|}
\hline consensus & the consensus nucleotide calculated as the nucleotide with highest count multiple nucleotides returned in a tie \\
\hline coverage & the number of reads covering this position \\
\hline non_ref_count & the number of reads that disagree with the reference nucleotide \\
\hline non_ref_count_hash & $\begin{array}{l}\text { a Hash with } A, T, G \text { and } C \text { as keys and the number each nucleotide appears in the pileup when that nucleotide is not } \\
\text { the reference }\end{array}$ \\
\hline pos & the position in the reference sequence that this pileup represents \\
\hline read_bases & the read nucleotides covering this position \\
\hline read_quals & the quality scores of the read nucleotides covering this position \\
\hline ref_base & the reference sequence nucleotide \\
\hline ref_count & the number of times the reference nucleotide appears in the read nucleotides covering this position \\
\hline ref_name & the name of the reference sequence \\
\hline ar1, ar2, ar3 & the allele calls from pileup \\
\hline consensus $^{1}$ & the consensus of the reads according to SAMtools method of calculation \\
\hline consensus_quality $^{1}$ & the quality score of the consensus according to SAMtools method of calculation \\
\hline rms_mapq $^{1}$ & the root mean square mapping quality at the position \\
\hline snp_quality ${ }^{1}$ & the SNP quality at the position \\
\hline
\end{tabular}

${ }^{1}$ ten column format only.

sam. index_stats

\# returns $\{$ "chr_1" $=>\{:$ length $=>69930$, : mapped_reads $=>1000$, \}

\section{Retrieving reference sequence}

Retrieving the reference can only be done if the reference has been loaded, which isn't done automatically in order to save memory. Reference need only be loaded once, and is accessed using reference name, start, end in 1-based coordinates. A standard Ruby String object is returned. In this example a 500 nucleotide region from the start of the sequence is returned.

bam. load_reference

seq = bam.fetch_reference ("Chr1", 1, 500 )

\section{Retrieving alignments in a region}

Alignments in a region of interest can be obtained one at a time by giving the region to the fetch() function.

bam.fetch("Chr1", 3000, 4000). each do | alignment |

puts alignment.qname \#do something

with the alignment object

end

\section{Get a summary of coverage in a region}

It is easy to get the total depth of reads at a given position, the chromosome_coverage function is used. This differs

from the previous functions in that a start position and length (rather than end position) are passed to the function. An array of coverages is returned, eg $[26,26,27 \ldots]$. The first position in the array gives the depth of coverage at the given start position in the genome, the last position in the array gives the depth of coverage at the given start position plus the length given.

coverages = bam. chromosome_coverage

("Chr1", 3000, 1000)

Similarly, average (arithmetic mean) of coverage can be retrieved, also with start and length parameters

av_cov = bam.average_coverage ("Chr1", $3000,1000)$

\section{Getting pileup information}

Pileup format represents the coverage of reads over a single base in the reference. Getting a Pileup over a region is very easy. Note that this is done with mpileup and NOT the now deprecated and removed from SAMTools pileup function. Calling the mpileup method creates an iterator that yields a Pileup object for each base.

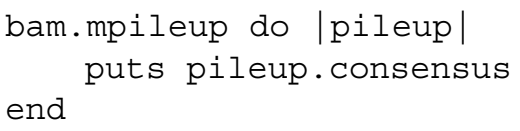

The mpileup function takes a range of parameters to allow SAMTools level filtering of reads and alignments. They are specified as key, value pairs. In this example a 
Table 4 SAMtools options recognised by the Bio::DB:Sam\#mpileup method and the symbols used to invoke them

\begin{tabular}{|c|c|c|c|c|}
\hline SAMTools option & description & short symbol & long symbol & default \\
\hline r & limit retrieval to a region & $: r$ & :region & all positions \\
\hline 6 & assume Illumina scaled quality scores & :six & :illumina_quals & FALSE \\
\hline A & count anomalous read pairs scores & :A & :count_anomalous & FALSE \\
\hline B & disable BAQ computation & :B & :no_baq & FALSE \\
\hline C & parameter for adjusting mapQ & : C & :adjust_mapq & 0 \\
\hline$d$ & max per-BAM depth to avoid excessive memory usage & $: d$ & :max_per_bam_depth & 250 \\
\hline $\mathrm{E}$ & extended BAQ for higher sensitivity but lower specificity & $: E$ & :extended_baq & FALSE \\
\hline $\mathrm{G}$ & exclude read groups listed in FILE & :G & :exclude_reads_file & FALSE \\
\hline I & list of positions (chr pos) or regions (BED) & $: 1$ & :list_of_positions & FALSE \\
\hline $\mathrm{M}$ & cap mapping quality at value & :M & :mapping_quality_cap & 60 \\
\hline $\mathrm{R}$ & ignore RG tags & $: R$ & :ignore_rg & FALSE \\
\hline q & skip alignments with mapping quality smaller than value & $: q$ & :min_mapping_quality & 0 \\
\hline Q & skip bases with base quality smaller than value & :Q & :imin_base_quality & 13 \\
\hline
\end{tabular}

region is specified by :r and a minimum per base quality score is specified by:Q.

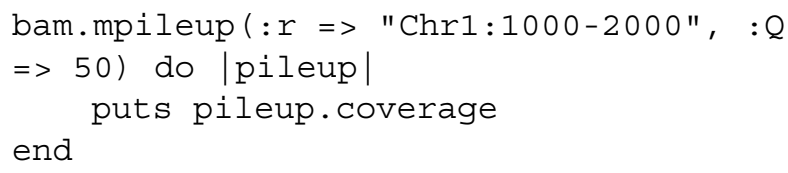

Not all the options SAMTools allows you to pass to mpileup are supported, those that cause mpileup to return Binary Variant Call Format (BCF) [13] are ignored. Specifically these are g,u,e,h,I,L,o,p. Table 4 lists the SAMTools flags supported and the symbols you can use to call them in the mpileup command.

\section{Conclusions}

Ruby is an easily written and understood high-level language, ideal for beginners or those wishing to develop analysis scripts and prototype applications in short timeframes. A major advantage of scripting in Ruby for biologists is the BioRuby project that provides a lot of classes and functionality for dealing with common biological data types and file formats. bio-samtools is a BioRuby plugin which extends the original BioRuby framework by providing a useful and flexible interface for Ruby coders who wish to have programmatical access to the data in BAM and SAM files without losing performance, the C API is very much quicker than a pure Ruby implementation would be and wrapping it provides the best of both languages. The interface we provide gives access to all the API components of the SAMtools core library libbam.so and extends with some useful high level methods. The open class system of Ruby means that the SAM class which encapsulates the functionality of SAMtools can easily be extended at run-time by the user. These features together mean that bio-samtools can be an extremely useful tool for scientists wishing to examine the results of next-generation sequencing alignments.

\section{Availability and requirements}

Project name: bio-samtools

Project home page: http://rubygems.org/gems/biosamtools

Operating systems: Linux and Mac OS X

Programming language: Ruby

Other requirements: none

License: as BioRuby

Any restrictions to use by non-academics: none

Competing interests

The authors declare that they have no competing interests.

\section{Authors' contributions}

RRG wrote the binding, tests, co-wrote the documentation and co-wrote the manuscript, RB created and organised the Gem and contributed to the binding and tests and co-wrote the manuscript and DM conceived of the binding, contributed to the binding and tests, tested the implementations with sample data and co-wrote the manuscript. RRG and RB contributed equally to this work. All authors read and approved the final manuscript.

\section{Acknowledgements}

RHRG and MC are supported by the BBSRC and DM is supported by The Gatsby Charitable Foundation. RHRG and RB contributed equally to this work.

\section{Author details}

${ }^{1}$ The Genome Analysis Centre, Norwich Research Park, Colney Lane, Norwich, NR4 7UH, UK. ${ }^{2}$ Istituto Nazionale Genetica Molecolare, Via F. Sforza 28, Milan 20122, Italy. ${ }^{3}$ The Sainsbury Laboratory, Norwich Research Park, Colney Lane, Norwich, NR4 7UH, UK.

Received: 26 April 2012 Accepted: 26 April 2012

Published: 28 May 2012

\section{References}

1. Li H, Durbin R: Fast and accurate short read alignment with Burrows-Wheeler transform. Bioinformatics 2009, 25(14):1754-60. [http://bioinformatics.oxfordjournals.org/cgi/content/full/25/14/1754? view=long\&pmid=19451168] 
2. Langmead B, Trapnell C, Pop M, Salzberg SL: Ultrafast and memory-efficient alignment of short DNA sequences to the human genome. Genome Bio/ 2009, 10(3):R25. [http://genomebiology.com/ content/10/3/R25]

3. Li R, Li Y, Kristiansen K, Wang J: SOAP: short oligonucleotide alignment program. Bioinformatics 2008, 24(5):713-4.

4. Novocraft. [http://www.novocraft.com]

5. Homer N, Merriman B, Nelson SF: BFAST: an alignment tool for large scale genome resequencing. PLOS ONE 2009, 4(11):e7767.

6. Li H, Handsaker B, Wysoker A, Fennell T, Ruan J, Homer N, Marth G, Abecasis G, Durbin R, Subgroup GPDP: The Sequence Alignment/Map format and SAMtools. Bioinformatics 2009, 25(16):2078-9. [http:// bioinformatics.oxfordjournals.org/cgi/content/full/25/16/2078]

7. Bio-SamTools. [http://search.cpan.org/lds/Bio-SamTools/]

8. pysam. [http://code.google.com/p/pysam/]

9. Picard. [http://picard.sourceforge.net/index.shtml]

10. BioRuby plugins. [http://bioruby.open-bio.org/wiki/Plugins]

11. Ruby Foreign Function Interface. [https://github.com/ffi/ff/wiki]

12. Pileup Format. [http://samtools.sourceforge.net/pileup.shtml]

13. Variant Call Format. [http://www.1000genomes.org/node/101]

doi:10.1186/1751-0473-7-6

Cite this article as: Ramirez-Gonzalez et al:: Bio-samtools: Ruby bindings for SAMtools, a library for accessing BAM files containing high-throughput sequence alignments. Source Code for Biology and Medicine 2012 7:6.

Submit your next manuscript to BioMed Central and take full advantage of:

- Convenient online submission

- Thorough peer review

- No space constraints or color figure charges

- Immediate publication on acceptance

- Inclusion in PubMed, CAS, Scopus and Google Scholar

- Research which is freely available for redistribution

Submit your manuscript at www.biomedcentral.com/submit
C Biomed Central 